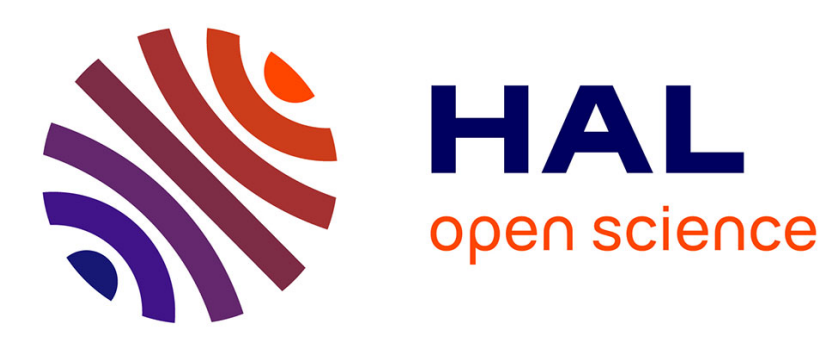

\title{
Essai d'amélioration par sélection de la qualité de carcasse du poulet de chair
}

F.H. Ricard, G. Marche, Elisabeth Le Bihan-Duval

\section{To cite this version:}

F.H. Ricard, G. Marche, Elisabeth Le Bihan-Duval. Essai d'amélioration par sélection de la qualité de carcasse du poulet de chair. Productions Animales, 1994, 7 (4), pp.253-261. hal-00896090

\section{HAL Id: hal-00896090 https://hal.science/hal-00896090}

Submitted on 1 Jan 1994

HAL is a multi-disciplinary open access archive for the deposit and dissemination of scientific research documents, whether they are published or not. The documents may come from teaching and research institutions in France or abroad, or from public or private research centers.
L'archive ouverte pluridisciplinaire HAL, est destinée au dépôt et à la diffusion de documents scientifiques de niveau recherche, publiés ou non, émanant des établissements d'enseignement et de recherche français ou étrangers, des laboratoires publics ou privés. 
INRA Prod. Anim., 1994,7 (4), $253-261$
F.H. RICARD, G. MARCHE,

E. LE BIHAN-DUVAL

INRA Station de Recherches Avicoles 37380 Nouzilly
Essai

d'amélioration par sélection de la qualité de carcasse du poulet de chair

La qualité de la carcasse du poulet est un sujet complexe qui comporte de nombreux éléments : conformation de l'animal, absence de défauts de présentation, engraissement, composition anatomique de la carcasse, ainsi que les aspects gustatif, fonctionnel et bactériologique de la viande. L'amélioration simultanée des composantes de la qualité est donc difficile à envisager de façon globale.

L'amélioration de deux des aspects a été entreprise par un programme de sélection sur 8 générations : nous avons cherché à diminuer l'état d'engraissement et à augmenter la partie la plus recherchée de la carcasse, les muscles pectoraux, tout en augmentant la vitesse de croissance. Cette expérience, réalisée sur une lignée synthétique de poulets à croissance rapide, a donné des résultats positifs très intéressants.

\section{Résumé}

Un problème important auquel sont confrontés tous les sélectionneurs de poulets de chair est d'améliorer en même temps la vitesse de croissance et les caractéristiques de

Une expérience d'amélioration de la qualité des carcasses par les techniques classiques de la sélection a été réalisée pendant 8 générations sur une lignée synthétique de poulets de chair à croissance rapide. La lignée de départ a été obtenue par croisements entre plusieurs souches commerciales et expérimentales et a ensuite été maintenue sans sélection orientée comme lignée "témoin". La lignée "qualité" était sélectionnée pour donner des poulets ayant un faible état d'engraissement et un fort développement des muscles perctoraux tout en étant aussi lourds que possible.

En raison des corrélations existant entre les caractères sélectionnés, la diminution de l'engraissement a été plus longue à obtenir que l'augmentation du rendement en muscles pectoraux. Mais les résultats sont encourageants : à la huitième génération de sélection, les poulets de la lignée "qualité" étaient environ $8 \%$ plus lourds que ceux de la lignée "témoin", les dépôts gras abdominaux étaient réduits de $21 \%$ et l'ensemble des muscles pectoraux était augmenté de $11 \%$.

Comparés aux témoins, les poulets de la lignée "qualitê" présentaient une meilleure conformation (angle de poitrine), un gésier un peu moins développé et une plus forte proportion des parties nobles de la carcasse (muscles grand et petits pectoraux, pilons, ailes). Les corrélations entre éléments de la carcasse, relativement homogènes à la huitième génération, ont montré que la sélection n'avait pas modifié sensiblement la structure anatomique de la carcasse. qualité des carcasses. L'amélioration de la vitesse de croissance s'est faite de façon constante depuis l'avant-guerre, où un poids vif de $2 \mathrm{~kg}$ n'était pas obtenu avant l'âge de 5 ou 6 mois. Entre 1962 et 1985 , le poids vif à 7 semaines a augmenté en moyenne de 45 grammes par an, d'après les testages réalisés à la Station de Ploufragan (L'Hospitalier et al 1986). Le poids vif moyen de $2 \mathrm{~kg}$ était obtenu vers l'âge de 7 semaines en 1980, alors qu'il fallait moins de 6 semaines en 1993.

Parallèlement, le sélectionneur doit limiter les défauts de carcasse de ses poulets (ampoules au bréchet, problèmes locomoteurs, par exemple) et diminuer l'engraissement de la carcasse, fortement corrélé au poids des dépôts gras abdominaux chez le poulet (Delpech et Ricard 1965). D'autre part, il doit augmenter le rendement en viande, en particulier le pourcentage des filets (partie de la carcasse qui a la valeur marchande la plus forte). Mais la difficulté tient au fait que la vitesse de croissance et les caractéristiques de qualité des carcasses sont souvent corrélées négativement entr'elles. Les analyses génétiques réalisées sur le poulet ont montré que les dépôts gras abdominaux présentaient une 
valeur d'héritabilité élevée (Ricard et Rouvier 1967, Ricard 1974, Becker et al 1984), qu'une sélection directe sur ce caractère était possible et qu'elle donnait des résultats positifs (Leclercq et al 1980). La lignée "maigre" de Leclercq donnait des poulets ayant une bonne qualité de carcasse, avec un meilleur rendement en viande et une viande un peu plus ferme associée à une flaveur plus développée (Ricard et Touraille 1988). De leur côté, Kazakov et al (1984) ont montré qu'il était possible de sélectionner des poulets à croissance rapide, ayant une carcasse maigre et une bonne teneur en viande (notion anglo-saxonne de "fleshing", qu'on peut traduire par "viandosité").

Un programme de sélection expérimentale a été mis en place en 1985 dans notre laboratoire pour essayer d'obtenir des poulets ayant à la fois une croissance rapide, une carcasse maigre et un bon rendement en muscles pectoraux. Une population de base a d'abord été obtenue, entre 1985 et 1987, à partir de croisements entre plusieurs souches commerciales et expérimentales. La génération de départ (G0) est née en mai 1987. Huit générations de sélection ont été obtenues ensuite (G1 à G8) et les principaux résultats sont présentés ici. A chaque génération, la lignée sélectionnée, dite "qualité", était comparée à la lignée "témoin" contemporaine, reproduite sans sélection orientée.

\section{1 / Conditions expérimentales}

\section{1 / Obtention des poussins}

La population de base a été obtenue à partir de croisements entre plusieurs souches commerciales (firmes ISA et Hubbard) et des lignées expérimentales de la Station de Recherches Avicoles de l'INRA. La figure 1 donne le schéma des croisements d'obtention de la lignée de départ (G0 obtenue en mai 1987) et les dates d'éclosion des différentes générations de sélection.

Les souches "Cornish" et "White-Rock" étaient sélectionnées par les firmes Hubbard et ISA pour la production de lignées commerciales de type "mâle-chair" ou "femelle-chair". La lignée expérimentale "White-Rock/SRA" avait été sélectionnée pour donner des poulets à forte glycémie basale chez qui les dépôts gras abdominaux étaient légèrement mais significativement réduits (Leclercq et al 1987). La lignée expérimentale "Beige/SRA", ou Beige de Nouzilly, avait été sélectionnée par J.P. Boyer sur un ensemble de caractéristiques zootechniques et avait une croissance proche d'une souche commerciale de type "femelle-chair". La lignée expérimentale "Maigre/SRA" correspondait à la lignée sélectionnée à la Station de Recherches Avicoles pour donner des poulets ayant des dépôts gras abdominaux très peu développés (Leclercq 1988). A la génération de

Figure 1. Schéma des croisements d'obtention de la lignée de départ (GO) et dates d'éclosion des différentes générations (ISA : Institut de Sélection Animale, SRA : Station de Recherches Avicoles, INRA).

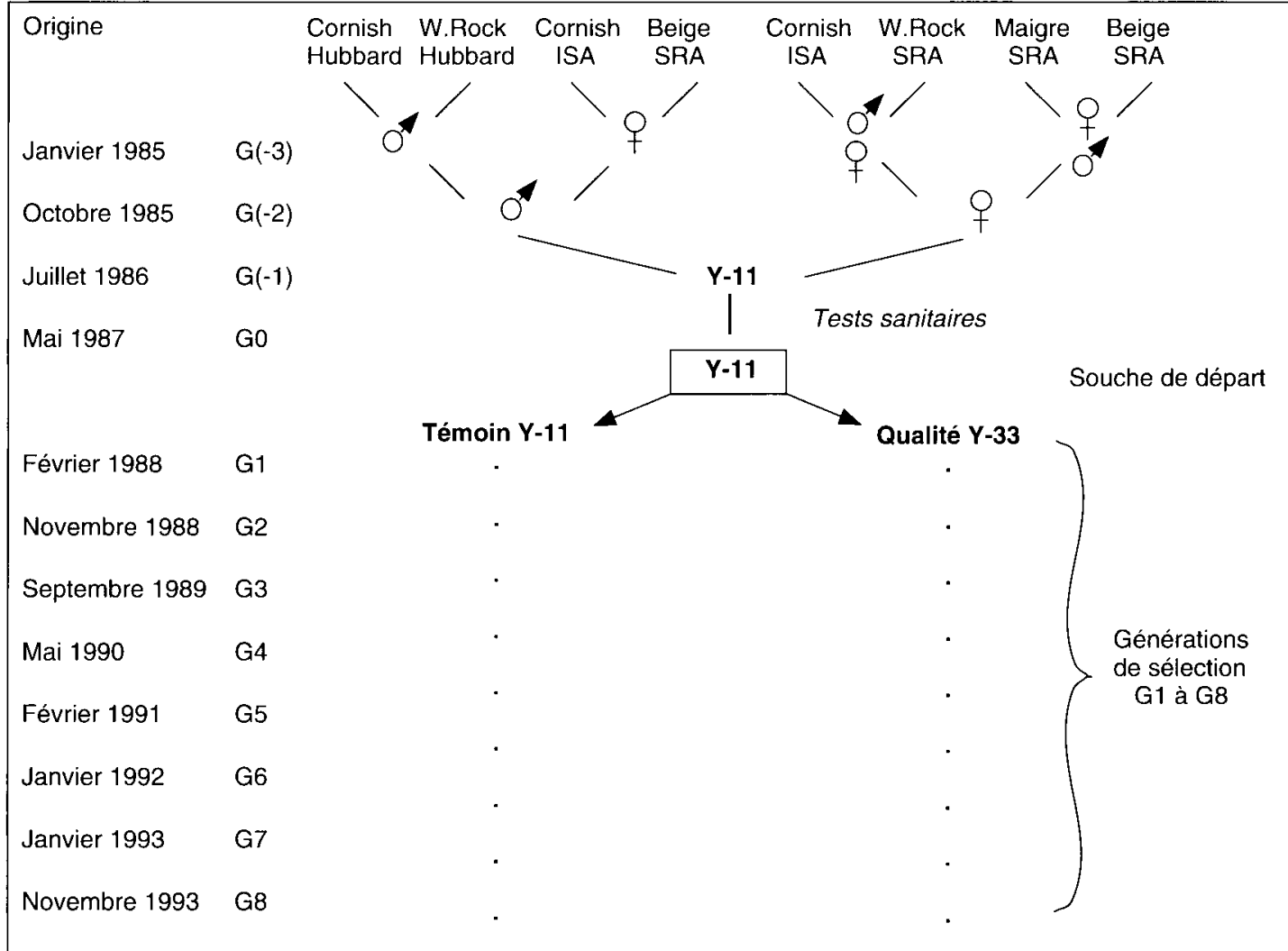


départ (G0), chaque poulet possédait $3 / 8 \mathrm{de}$ gènes "Cornish", 2/8 de gènes "White-Rock", $2 / 8$ de gènes "Beige" et $1 / 8$ de gènes "Maigre".

Un effort important a été fait, en collaboration avec la Station de Pathologie Aviaire de Nouzilly, pour rendre le statut sanitaire de ces lignées aussi bon que possible. A chaque génération d'homogénéisation, les candidates reproductrices ont été testées pour détecter la présence éventuelle de virus leucosique (protéine virale P.27) par la méthode des tests ELISA sur albumen. Des tests sérologiques (séro-agglutinations) ont également été réalisés chez tous les candidats reproducteurs pour rechercher d'autres anticorps (Salmonella pullorum, Mycoplasma gallisepticum et synoviae, Adenovirus, Pseudoadenovirus, Reovirus). Les reproducteurs réellement retenus pour donner la génération de départ (G0) étaient tous indemmes de leucose aviaire et de pullorose et partiellement indemmes pour les autres anticorps testés.

La lignée "témoin" (nom de code Y-11) a été reproduite sans sélection orientée, selon la technique de King et al (1959) : choix au hasard d'un fils par père et d'une fille par mère, puis accouplements en évitant les mariages frère $\mathrm{x}$ sœur et demi-frère $\mathrm{x}$ demisœur. La lignée "qualité" (nom de code Y-33) était reproduite à partir de tests sur collatéraux : mise en élevage d'un lot spécial de poussins puis abattages à l'âge de 6 semaines et dissection de 4 à 5 poulets par famille, les valeurs brutes étant transformées en écartsréduits, séparément pour chaque sexe. Les moyennes de famille ont ensuite été calculées, sexes mélangés, pour le pourcentage des dépôts gras abdominaux et celui des muscles pectoraux. Cette technique permettait de choisir les familles ayant à la fois un faible engraissement et des muscles pectoraux développés. Ensuite, dans le lot sélectionné, les candidats reproducteurs retenus étaient les poulets appartenant à ces familles qui avaient la plus forte vitesse de croissance (et, dans les deux premières générations, le meilleur angle de poitrine).

A chaque génération de sélection et pour chaque lignée, 15 à 18 pères et 3 à 5 mères par père étaient utilisés comme reproducteurs. Les poussins du cheptel "sélection" (250 à 350 par lignée et par génération) étaient obtenus en un seul lot d'éclosion (ramassage des oufs sur 2 semaines), ils étaient élevés ensemble, au sol dans la même case de poussinière, sur litière de copeaux de bois. Ils recevaient la même alimentation (tableau 1) et le même programme de prophylaxie. Il convient toutefois de signaler qu'à la sixième génération de sélection les poulets des 2 lignées ont été élevés dans 2 cases de poussinière différentes.

La croissance était contrôlée par pesée individuelle de tous les animaux (à jeun) aux âges de 3,6 , et 7 semaines. Après la pesée de 7 semaines, les animaux étaient rationnés afin d'éviter un poids trop élevé à l'âge adulte, synonyme d'engraissement excessif, d'apparition de problèmes locomoteurs et, finalement, de mauvaises performances de reproduction.
Tableau 1. Caractéristiques des aliments utilisés (moyennes calculées).

\begin{tabular}{|lccccc|}
\hline & \multicolumn{5}{c|}{ Période d'élevage } \\
\cline { 2 - 6 } & $\mathbf{0 * 3 ~ s e m ~}$ & $\mathbf{3 - 7}$ sem & $\mathbf{7 - 2 0}$ sem & poules & coqs \\
\hline Energie métab. $(\mathrm{kJ} / \mathrm{g}))$ & 12,7 & 12,5 & 11,9 & 11,7 & 12,8 \\
Protéines brutes $(\%)$ & 22,1 & 19,0 & 15,6 & 16,5 & 12,6 \\
Lysine (\%) & 1,16 & 0,91 & 0,59 & 0,73 & 0,46 \\
Ac. aminés soufrés (\%) & 0,86 & 0,70 & 0,54 & 0,30 & 0,48 \\
Phosphore assimil. (\%) & 0,46 & 0,37 & 0,33 & 0,34 & 0,21 \\
Calcium (\%) & 0,96 & 0,89 & 0,76 & 3,40 & 1,21 \\
Présentation & granulé & granulé & farine & farine & farine \\
& $2,5 \mathrm{~mm}$ & $4,0 \mathrm{~mm}$ & & & \\
\hline
\end{tabular}

La quantité d'aliment était limitée à 70 grammes par jour et par sujet à l'âge de 7 semaine puis augmentée jusqu'à un maximum de 110 grammes pour les coqs (à l'âge de 20 semaines) et de 130 grammes pour les poules (à l'âge de 22 semaines). La durée d'éclairement du poulailler était également limitée : éclairage continu durant les 3 premiers jours, puis 14 heures de lumière par jour jusqu'à l'âge de 7 semaines, puis 8 heures par jour jusqu'à l'âge de 16 semaines, puis augmentation d'une heure chaque semaine jusqu'à 15 heures par jour à l'âge de 22 semaines.

Les animaux conservés pour la reproduction étaient transférés vers l'âge de 20 semaines dans des cages individuelles et les poussins étaient obtenus par insémination artificielle, selon un système de reproduction pédigrée.

\section{2 / Comparaison des lignées pour les caractéristiques de carcasse}

Un lot spécial de poulets, élevés dans des conditions comparables au lot "sélection", était généralement utilisé à chacune des générations pour ces comparaisons. Un échantillon d'une trentaine de poulets par sexe et par lignée, représentant la répartition de l'ensemble des poids vifs, était abattu à l'âge de 7 semaines, après un jeûne de 16 heures. Le poids vif avant abattage était obtenu ainsi que l'angle de poitrine mesuré sur la carcasse plumée.

Une technique de dissection voisine de celle proposée par le Groupe de travail $n^{\circ} 5$ de la W.P.S.A. (1984) a permis d'obtenir la pesée des éléments suivants : ensemble des dépôts gras abdominaux (gras pariétal + gras entourant le gésier et le ventricule succenturié), gésier musculaire, foie (sans la vésicule biliaire), carcasse semi-éviscérée (avec cou, trachée artère, poumons, cœur, reins, gonades), ensemble des muscles pectoraux (Pectoralis superficialis, $P$. profundus, P. supracoracoideus, et partie externe des Coracobrachialis ventralis, selon la nomenclature de Chamberlain 1943), cuisses, pilons et ailes. On a ensuite calculé le pourcentage de chacun de ces éléments par rapport au poids vif avant abattage.

Les paramètres statistiques de chaque variable ont été calculés et les moyennes ont été comparées par une analyse classique de variance à 2 niveaux (lignée x sexe). Les corrélations phénotypiques entre les variables ont également été calculées. 


\section{2 / Résultats}

Le poids vif moyen de la lignée qualité est plus élevé dès la première génération de sélection.

\section{1 / Vitesse de croissance}

Les données concernant la vitesse de croissance sont rassemblées dans le tableau 2 qui donne les poids vifs aux âges de 3,6 et 7 semaines par lignée et par sexe. Les différences entre lignées sont presque toujours hautement significatives.

L'évolution en fonction des générations est illustrée par la figure 2 , qui donne le poids vif à 6 semaines de chaque lignée pour la moyenne des 2 sexes. On peut voir que la lignée "qualité" est au dessus de la lignée "témoin" dès la première génération de sélection. Les deux lignées ont ensuite une évolution comparable, la lignée "qualité" étant supérieure d'environ $6 \%$ à la première et de $8 \%$ à la huitième génération. On peut voir également qu'il existe des variations non régulières entre générations pour chacune des 2 lignées, même si on observe une augmentation générale du poids vif

Tableau 2. Mesures de croissance (poids vifs en grammes) pour chaque génération de sélection

\begin{tabular}{|c|c|c|c|c|c|c|}
\hline \multirow[b]{2}{*}{ lignée } & \multicolumn{3}{|c|}{ Mâles } & \multicolumn{3}{|c|}{ Femelles } \\
\hline & témoin & & qualité & témoin & & Ialité \\
\hline \multicolumn{7}{|l|}{ Poids vif à 3 semaines } \\
\hline G0 & 453 & & & 423 & & \\
\hline G1 & 444 & $* *$ & 484 & 418 & $* *$ & 448 \\
\hline G2 & 496 & $* *$ & 529 & 450 & *** & 481 \\
\hline G3 & 481 & $* *$ & 503 & 456 & $\mathrm{~ns}$ & 465 \\
\hline G4 & 453 & $* *$ & 480 & 415 & $* *$ & 446 \\
\hline G5 & 507 & $* *$ & 526 & 465 & $* *$ & 480 \\
\hline G6 & 517 & (1) & 517 & 477 & (1) & 476 \\
\hline G7 & 593 & $* * *$ & 625 & 529 & $* *$ & 562 \\
\hline G8 & 601 & $* *$ & 641 & 535 & $* *$ & 570 \\
\hline \multicolumn{7}{|l|}{ Poids vif à 6 semaines } \\
\hline G0 & 1429 & & & 1246 & & \\
\hline G1 & 1477 & $* *$ & 1566 & 1252 & $* * *$ & 1330 \\
\hline G2 & 1672 & $* *$ & 1780 & 1397 & $* *$ & 1499 \\
\hline G3 & 1575 & $* *$ & 1667 & 1361 & $*$ & 1406 \\
\hline G4 & 1559 & $* *$ & 1617 & 1311 & $* *$ & 1389 \\
\hline G5 & 1723 & $* *$ & 1807 & 1445 & $* *$ & 1513 \\
\hline G6 & 1686 & (1) & 1782 & 1382 & (1) & 1499 \\
\hline G7 & 1831 & $* *$ & 1977 & 1490 & $* *$ & 1619 \\
\hline G8 & 1854 & $* *$ & 1977 & 1514 & $* *$ & 1643 \\
\hline \multicolumn{7}{|l|}{ Poids vif à 7 semaines } \\
\hline G0 & 1824 & & & 1554 & & \\
\hline G1 & 2019 & $* *$ & 2121 & 1670 & $* *$ & 1773 \\
\hline G2 & 2126 & $* *$ & 2258 & 1717 & $* *$ & 1852 \\
\hline G3 & 2030 & $* *$ & 2154 & 1709 & $* *$ & 1792 \\
\hline G4 & 1902 & $* *$ & 1959 & 1578 & $* *$ & 1669 \\
\hline G5 & 2045 & $* *$ & 2132 & 1689 & $* *$ & 1772 \\
\hline G6 & 2178 & (1) & 2162 & 1770 & (1) & 1789 \\
\hline G7 & 2301 & $* *$ & 2463 & 1842 & $* *$ & 1988 \\
\hline G8 & 2289 & $* *$ & 2448 & 1849 & $* *$ & 1994 \\
\hline
\end{tabular}

Signification des différences entre lignées: $\mathrm{ns}=$ différence non significative $(\mathrm{P}>0,05),{ }^{*}=\mathrm{P}<0,05$ et $* *=\mathrm{P}<0,01$

(1) à la sixième génération, les deux lignées ont été élevées dans 2 cases de poussinière différentes.
Figure 2. Evolution du poids vif à 6 semaines (moyenne des 2 sexes) en fonction des générations de sélection.

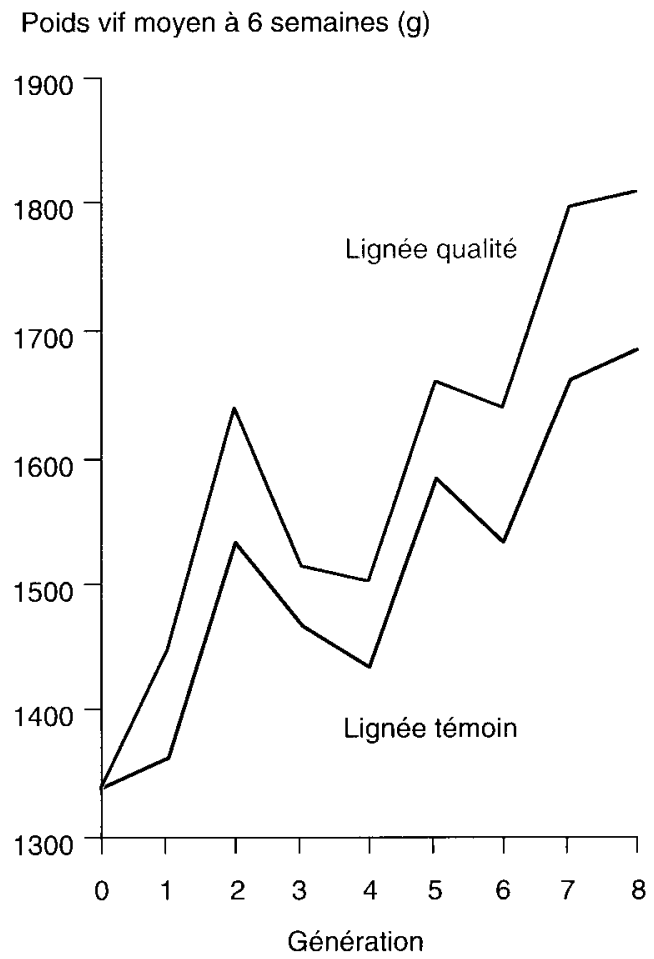

entre les générations 1 et 8 . Or, si la lignée "qualité" est sélectionnée (avec une pression de sélection modérée) pour augmenter la vitesse de croissance, le pool génétique de la lignée "témoin" reste théoriquement constant d'une génération à l'autre. Nous attribuons donc ces variations à une modification non contrôlée des conditions expérimentales. Ainsi, les animaux ne sont pas nés à la même saison, la composition des matières premières utilisées dans l'alimentation et les conditions climatiques peuvent changer d'une année à l'autre, les cases de poussinière utilisées ont varié en fonction de la place disponible au moment de la mise en place des poussins, les bâtiments utilisés ont été modifiés pour améliorer les conditions d'élevage (en particulier la ventilation), et le plan de prophylaxie a été adapté en cours d'expérience pour tenir compte des connaissances nouvelles en matière d'art vétérinaire.

\section{2 / Réponses directes à la sélection}

Elles sont résumées dans le tableau 3 qui donne les valeurs relatives de la lignée "qualité" par rapport à la lignée "témoin", pour les pourcentages des dépôts gras abdominaux et de l'ensemble des muscles pectoraux mesurés à l'âge de 7 semaines.

Pour le gras abdominal, la modification n'est apparue significative qu'à la sixième génération pour les mâles et à la septième pour les femelles. Au contraire, la modification est apparue significative dès la troisième génération pour les muscles pectoraux. Ce résultat peut s'expliquer par les corrélations entre caractères dont le tableau 4 donne les valeurs 
pour la lignée témoin au début et à la fin de l'expérience. On peut voir que le poids vif est corrélé positivement à la fois avec le pourcentage de gras abdominal et le pourcentage de muscles pectoraux. Entre ces deux pourcentages, la corrélation n'est pas significative et a tendance à être négative. Il apparaît donc biologiquement plus difficile de diminuer l'engraissement que d'améliorer le rendement en viande si on veut augmenter en même temps la vitesse de croissance. Mais cette remarque peut également être liée au stade physiologique de la croissance auquel on se place (à l'âge de 7 semaines, les poulets des lignées "chair" ont un poids vif de l'ordre du tiers du poids adulte d'animaux qui n'auraient pas été rationnés).

\section{3 / Réponses corrélées}

Dans le tableau 5 sont indiqués les résultats de dissection observés à la huitième génération (dernière génération étudiée). Les variables autres que le poids vif ainsi que les pourcentages de gras abdominal et de muscles pectoraux peuvent être considérées comme des caractères directement corrélés. Les interactions lignée $\mathrm{x}$ sexe ne sont pas significatives, à l'exception du poids vif, du poids de l'ensemble des muscles pectoraux et du pourcentage de

Tableau 3. Valeurs relatives de la lignée "qualité" pour l'engraissement et le pourcentage des muscles pectoraux, mesurés à l'âge de 7 semaines $(100=$ valeur de la lignée "témoin") pour les générations successives.

\begin{tabular}{|ccc|cc|}
\hline & \multicolumn{2}{c|}{ Mâles } & \multicolumn{2}{c|}{ Femelles } \\
& \% gras abdo & \% M. pector. & \% gras abdo & \% M. pector. \\
\hline G1 & $106,1 \mathrm{~ns}$ & $104,2 \mathrm{~ns}$ & $100,0 \mathrm{~ns}$ & $100,7 \mathrm{~ns}$ \\
G2 & $92,4 \mathrm{~ns}$ & $102,3 \mathrm{~ns}$ & $105,6 \mathrm{~ns}$ & $103,2 \mathrm{~ns}$ \\
G3 & $96,9 \mathrm{~ns}$ & $105,1^{*}$ & $101,8 \mathrm{~ns}$ & $105,0^{*}$ \\
G4 & $95,2 \mathrm{~ns}$ & $106,1^{* *}$ & non mesuré & non mesuré \\
G5 & $95,4 \mathrm{~ns}$ & $107,6^{* *}$ & $100,5 \mathrm{~ns}$ & $107,6 * *$ \\
G6 & $84,8^{* *}$ & $108,9^{* *}$ & $92,3 \mathrm{~ns}$ & $110,5 * *$ \\
G7 & $77,3^{* *}$ & $111,6^{* *}$ & $87,7^{* *}$ & $112,6 * *$ \\
G8 & $83,9^{* *}$ & $110,5^{* *}$ & $75,3^{* *}$ & $112,1 * *$ \\
\hline
\end{tabular}

Signification des différences entre les 2 lignées : $\mathrm{ns}=$ non significatif, ${ }^{*}=\mathrm{P}<0,05 * *=\mathrm{P}<0,01$.

gras abdominal, où les différences entre lignées sont relativement plus faibles chez les mâles que chez les femelles.

La comparaison globale des deux lignées fait apparaître des résultats significativement différents, sauf pour le pourcentage de foie, de cuisses, et pour la part des muscles "autres pectoraux" dans l'ensemble des muscles pectoraux. En ce qui concerne les valeurs brutes, les dépôts gras abdominaux sont plus élevés dans
La proportion de muscles pectoraux a augmenté sigifícativement dès la $3^{\text {eme }}$ génération alors que les dépôts gras abdominaux n'ont diminué $q u$ 'à la G $^{\text {ème }}$.

Tableau 4. Caractéristiques de croissance, d'engraissement et de développement musculaire observées dans la lignée "témoin" au début et à la fin de l'expérience de sélection, à l'âge de 7 semaines ( $M$ : mâles, $F$ : femelles).

\begin{tabular}{|c|c|c|c|c|c|}
\hline \multirow{2}{*}{$\begin{array}{l}\text { Génération } \\
\text { Effectif }\end{array}$} & \multirow{2}{*}{$\begin{array}{l}G(-1) \\
60 M\end{array}$} & \multicolumn{2}{|c|}{ G0 } & \multicolumn{2}{|c|}{ G8 } \\
\hline & & $47 \mathrm{M}$ & $27 \mathrm{~F}$ & $34 \mathbf{M}$ & $30 \mathrm{~F}$ \\
\hline \multicolumn{6}{|l|}{ Moyennes } \\
\hline Poids vif (g) & 1724 & 1780 & 1453 & 2439 & 1891 \\
\hline Angle (grades) & 84,5 & 83,3 & 85,6 & 97,7 & 95,7 \\
\hline Gras abdom. (g) & 48,2 & 51,8 & 46,9 & 74,7 & 75,4 \\
\hline Musc. pect. (g) & 203,7 & 199,7 & 178,8 & 326,3 & 255,7 \\
\hline$\%$ Gras abdom. & 2,8 & 2,9 & 3,2 & 3,1 & 4,0 \\
\hline$\%$ M. pectoraux & 11,8 & 11,2 & 11,8 & 13,4 & 13,5 \\
\hline \multicolumn{6}{|c|}{ Corrélations phénotypiques entre } \\
\hline Poids gras abdo et & & & & & \\
\hline P. Musc. pect. & $0,57^{*}$ & $0,57^{*}$ & 0,30 & 0,27 & $0,55^{*}$ \\
\hline \multicolumn{6}{|l|}{ Poids vifet } \\
\hline Angle poitrine & 0,32 & 0,22 & 0,30 & 0,23 & 0,19 \\
\hline$\%$ Gras abdo. & $0,47^{*}$ & $0,30^{*}$ & 0,35 & 0,03 & $0,47^{*}$ \\
\hline \% Musc. pect. & $0,35^{*}$ & 0,17 & $0,39 *$ & 0,26 & 0,29 \\
\hline \multicolumn{6}{|l|}{ Angle poitrine et } \\
\hline \% Gras abdo. & 0,15 & $0,41^{*}$ & $0,43^{*}$ & 0,04 & 0,20 \\
\hline$\%$ Musc. pect. & $0,31^{*}$ & $0,39^{*}$ & 0,03 & $0,43^{*}$ & $0,39 *$ \\
\hline $\begin{array}{l}\text { \% Gras abdominal et } \\
\% \text { Musc. pect. }\end{array}$ & $-0,05$ & 0,23 & $-0,30$ & $-0,16$ & $-0,04$ \\
\hline
\end{tabular}

* corrélation significativement différente de zéro $(\mathrm{P}<0,05)$, compte tenu des effectifs étudiés.

La génération G(-1) correspond aux animaux nés en 1986 qui ont fourni les reproducteurs de la génération de départ (G0) née en mai 1987. G8 correspond aux animaux de la lignée "témoin" à la huitième génération de sélection. 
la lignée "témoin". En ce qui concerne les pourcentages par rapport au poids vif, on voit que le gras abdominal ainsi que le gésier sont significativement plus développés dans la lignée "témoin". Pour les autres valeurs brutes et pourcentages, la lignée "qualité" présente des valeurs significativement plus élevées. En ce qui concerne la répartition du total des muscles pectoraux, le grand pectoral est relativement plus développé et les petits pectoraux sont relativement moins développés dans la lignée "qualité", comparativement à la lignée "témoin".

Le tableau 6 regroupe les valeurs des principales corrélations phénotypiques observées à la huitième génération de sélection entre, d'une part, le poids vif ou l'angle de poitrine et, d'autre part, le poids des éléments de la carcasse et les principaux rendements, séparément pour chacune des 2 lignées (moyennes observées chez les mâles et chez les femelles).
Le poids vif est corrélé significativement et positivement avec le poids de tous les éléments de la carcasse, à l'exception du gésier dans la lignée "qualité". C'est rarement le cas avec les mesures de rendement, en dehors du gras abdominal, du muscle grand pectoral et des ailes pour la lignée "témoin", et de la carcasse pour la lignée "qualité". La corrélation entre le poids vif et l'angle de poitrine est relativement faible (en moyenne 0,21 pour la lignée "témoin" et 0,26 pour la lignée "qualité"). Avec l'angle de poitrine, le tableau 6 montre que les corrélations sont faibles et rarement significatives, sauf en ce qui concerne le poids et le pourcentage du muscle grand pectoral dans les 2 lignées, ainsi que le poids de la carcasse et des cuisses dans la lignée "qualité".

Les principales corrélations observées à la huitième génération de sélection entre mesures de rendement sont regroupées dans le tableau 7. L'engraissement, mesuré par le

Tableau 5. Valeurs moyennes et analyses de variance à 2 niveaux (lignée $x$ sexe) des caractéristiques de carcasse observées à la huitième génération de sélection, à l'âge de 7 semaines.

A la $8^{\text {ème }}$ génération, les 2 lignées sont significativement différentes pour la plupart des caractéristiques.

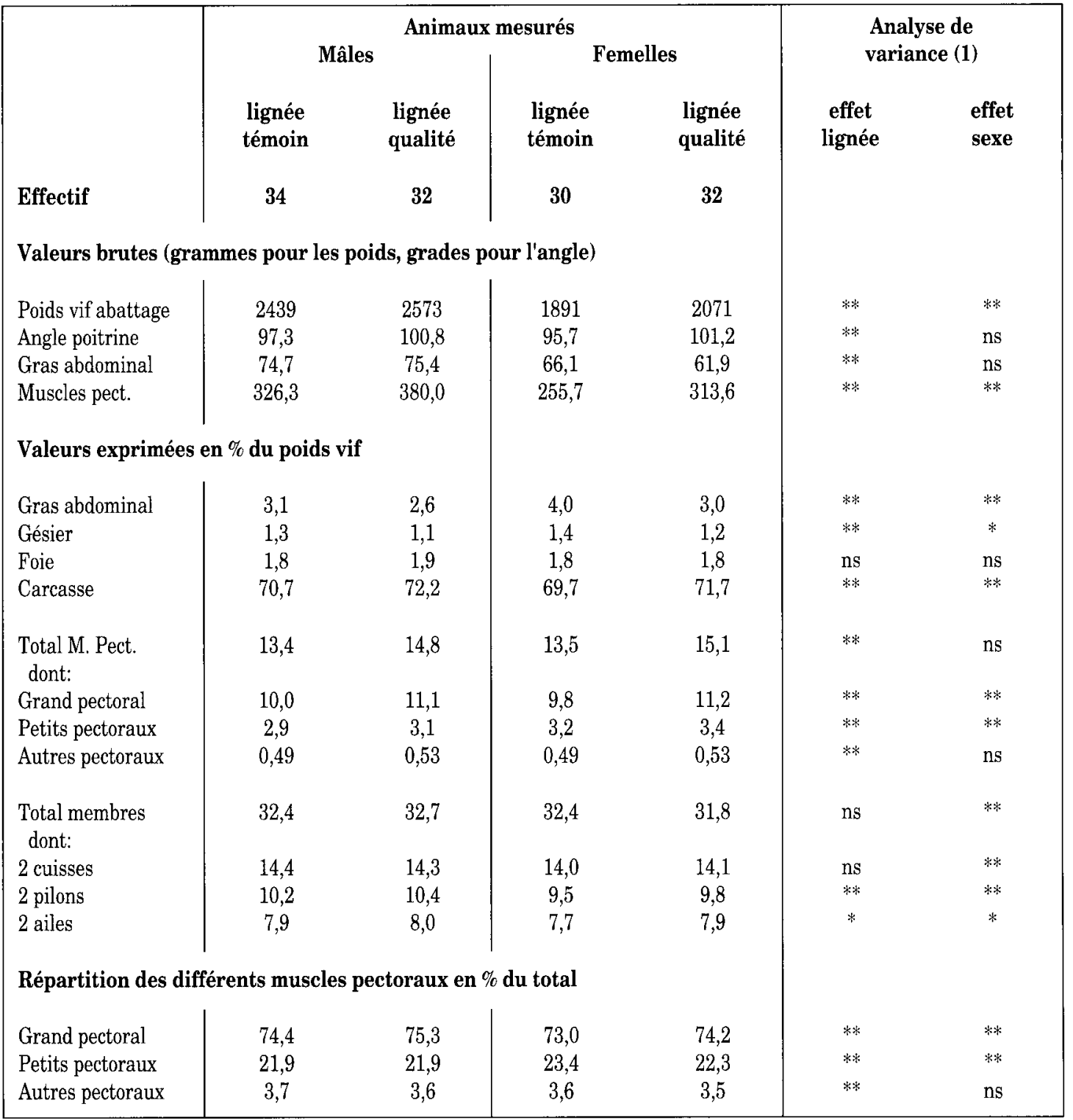

(1) test $\mathrm{F}: \mathrm{ns}=$ valeur non significative, ${ }^{*}=\mathrm{P}<0,05$ et ${ }^{* *}=\mathrm{P}<0,01$ 
pourcentage des dépôts gras abdominaux, est corrélé négativement au pourcentage des muscles pectoraux (valeurs significatives pour les muscles "autres pectoraux" dans les 2 lignées), et au pourcentage des ailes. Le rendement total des muscles pectoraux est naturellement corrélé significativement au pourcentage des différents muscles pectoraux, surtout le grand pectoral (valeur de la corrélation de l'ordre de 0,97 ). Il est également corrélé significativement au pourcentage de la carcasse éviscérée. Globalement, la corrélation entre les pourcentages du gras abdominal et des muscles pectoraux sont négatives : valeur de $-0,10$, non significative, pour la lignée "témoin" ( $P>0,05$ ), valeur de $-0,25$, significative, pour la lignée "qualité" $(\mathrm{P}<0,05)$.

\section{3 / Discussion}

Les données observées ne font apparaître qu'un gain de poids relativement faible $(+8 \%)$ dans la lignée "qualité" par rapport à la lignée "témoin". Ce résultat est dû au fait que la vitesse de croissance n'était pas le caractère prioritaire dans notre expérience de sélection qui était orientée d'abord sur l'engraissement et le développement musculaire. De plus, du fait de la corrélation positive entre le poids vif et le pourcentage des dépôts gras abdominaux, il est plus difficile d'obtenir à la fois une croissance élevée et un engraissement réduit. D'autre part, il ne faut pas oublier qu'il s'agit de lignées expérimentales portant sur des effectifs relativement faibles, si on les compare à ce qui se passe pour les principales lignées commerciales. Par rapport aux animaux élevés en France, les poulets de la lignée "témoin" ont environ une semaine de retard et ceux de la lignée "qualité" une demi-semaine de retard, si on se réfêre aux testages de Ploufragan réalisés en 1992, où le poids vif moyen à 41 jours était de 2156 grammes (Bougon et al 1993).

Quand on s'intéresse aux critères directement sélectionnés, on constate que la méthode utilisée a donné des résultats significatifs et intéressants pour les sélectionneurs de lignées de poulets de type "chair". A la huitième génération nous avons obtenu une diminution du pourcentage des dépôts gras abdominaux de $21 \%$ et une augmentation du pourcentage des muscles pectoraux de $11 \%$, en même temps qu'une augmentation du poids vif de $8 \%$, par comparaison avec la lignée "témoin". Ce résultat confirme donc pleinement les possibilités entrevues à la suite des travaux de Becker et al 1984, de Kazakov et al (1984) ou de Ricard et Touraille (1988). Il serait sans doute possible d'obtenir des résultats plus nets si on pouvait travailler sur des effectifs plus importants et donc avoir une pression de sélection plus forte que celle que nous pouvions obtenir dans nos conditions expérimentales.

La dissection des 2 types de poulets a fait apparaître de nombreuses différences significatives entre lignées. Les différences entre pourcentages des dépôts gras abdominaux et de l'ensemble des muscles pectoraux correspondent bien à ce qui était recherché. Le pour-
Tableau 6. Corrélations phénotypiques observées à la huitième génération de sélection entre, d'une part, le poids vif à l'abattage ou l'angle de poitrine mesuré sur la carcasse et, d'autre part, le poids des différents éléments de la carcasse et les rendements. Moyennes des 2 sexes pour chacune des 2 lignées.

\begin{tabular}{|c|c|c|c|c|}
\hline \multirow{2}{*}{ Lignée } & \multicolumn{2}{|c|}{ Avec le poids vif } & \multicolumn{2}{|c|}{ Avec l'angle } \\
\hline & témoin & qualité & témoin & qualité \\
\hline \multicolumn{5}{|c|}{ Poids bruts des différents éléments } \\
\hline Gésier & $0,25^{*}$ & $-0,07$ & $-0,14$ & $-0,15$ \\
\hline Foie & $0,41^{*}$ & $0,67^{*}$ & 0,01 & 0,09 \\
\hline Gras abdominal & $0,58^{*}$ & $0,36^{*}$ & 0,17 & 0,21 \\
\hline Carcasse totale & $0,97^{*}$ & $0,98^{*}$ & 0,24 & $0,28^{*}$ \\
\hline Total musc. pect. & $0,82^{*}$ & $0,77^{*}$ & $0,38^{*}$ & $0,33^{*}$ \\
\hline M. grand pectoral & $0,81^{*}$ & $0,75^{*}$ & $0,44^{*}$ & $0,35^{*}$ \\
\hline M. petits pectoraux & $0,78^{*}$ & $0,67 *$ & 0,19 & 0,21 \\
\hline Autres pectoraux & $0,70^{*}$ & $0,47^{*}$ & 0,08 & $-0,03$ \\
\hline Total membres & $0,93^{*}$ & $0,94^{*}$ & 0,14 & $0,26^{*}$ \\
\hline 2 cuisses & $0,89^{*}$ & $0,88^{*}$ & 0,11 & $0,26^{*}$ \\
\hline 2 pilons & $0,85^{*}$ & $0,83^{*}$ & 0,09 & 0,24 \\
\hline 2 ailes & $0,87^{*}$ & $0,83^{*}$ & 0,23 & 0,15 \\
\hline \multicolumn{5}{|c|}{ Rendements (en $\%$ du poids vif avant abattage) } \\
\hline \% gésier & $-0,12$ & $-0,37^{*}$ & $-0,22$ & $-0,23$ \\
\hline$\%$ foie & $-0,17$ & 0,08 & $-0,10$ & $-0,06$ \\
\hline$\%$ gras abdominal & $0,25^{*}$ & 0,10 & 0,12 & 0,17 \\
\hline$\%$ carcasse totale & 0,22 & $0,42^{*}$ & 0,19 & $0,25^{*}$ \\
\hline$\%$ ensemble pector. & $0,27^{*}$ & 0,07 & $0,41^{*}$ & 0,22 \\
\hline$\%$ grand pectoral & $0,29^{*}$ & 0,10 & $0,50^{*}$ & $0,27^{*}$ \\
\hline$\%$ petits pectoraux & 0,15 & $-0,04$ & 0,07 & 0,03 \\
\hline$\%$ autres pectoraux & $-0,02$ & $-0,03$ & $-0,09$ & $-0,19$ \\
\hline$\%$ cuisses & 0,13 & 0,17 & $-0,13$ & 0,13 \\
\hline$\%$ pilons & $-0,04$ & 0,07 & $-0,17$ & 0,06 \\
\hline$\%$ ailes & $-0,35^{*}$ & 0,00 & 0,02 & $-0,09$ \\
\hline
\end{tabular}

* corrélation significativement différente de zéro $(\mathrm{P}<0,05)$.

centage plus faible du gésier observé dans la lignée "qualité" peut être attribué à une allométrie négative entre le poids du gésier et le poids vif. Pour les autres rendements, la sélection réalisée donne des résultats favorables. En ce qui concerne la répartition des différents muscles pectoraux, la sélection a favorisé le muscle grand pectoral par rapport aux petits pectoraux. Il faudrait vérifier si la composition et la structure de ces muscles pectoraux ont été, ou non, modifiés.

Les corrélations observées sur les animaux de la huitième génération sont relativement homogènes entre les 2 types de lignées. Les valeurs entre angle de poitrine et poids des cuisses ou des pilons sont un peu plus fortes dans la lignée "qualité", les valeurs entre angle de poitrine et muscle grand pectoral sont un peu plus fortes dans la lignée "témoin", mais dans l'ensemble on peut dire que la sélection 
Tableau 7. Corrélations phénotypiques observées à la huitième génération de sélection entre les pourcentages de gras abdominal ou du total des muscles pectoraux avec les autres rendements (par rapport au poids vif). Moyennes des 2 sexes pour chacune des 2 lignées.

\begin{tabular}{|lcccc|}
\hline \multirow{2}{*}{ Lignée } & \multicolumn{2}{c}{ \% gras abdominal } & \multicolumn{2}{c|}{ \% ensemble pectoraux } \\
& témoin & qualité & témoin & qualité \\
\hline \% gésier & $-0,20$ & $-0,16$ & $-0,29^{*}$ & $-0,36^{*}$ \\
\% foie & $-0,06$ & $-0,01$ & $-0,19$ & $-0,45^{*}$ \\
\% carcasse totale & $-0,00$ & $-0,23$ & $0,54^{*}$ & $0,44^{*}$ \\
& & & & \\
\% total M. pect. & $-0,10$ & $-0,25^{*}$ & - & - \\
\% grand pectoral & $-0,09$ & $-0,24$ & $0,98^{*}$ & $0,97^{*}$ \\
\% petits pecto. & $-0,01$ & $-0,16$ & $0,80^{*}$ & $0,70^{*}$ \\
\% autres pecto. & $-0,47^{*}$ & $-0,39^{*}$ & $0,27^{*}$ & $0,30^{*}$ \\
& & & & \\
\% cuisses & 0,12 & $-0,01$ & $-0,06$ & $-0,27^{*}$ \\
\% pilons & $-0,20$ & $-0,17$ & $-0,04$ & $-0,14$ \\
\% ailes & $-0,30^{*}$ & $-0,60^{*}$ & 0,18 & 0,23 \\
\hline
\end{tabular}

* corrélation significativement différente de zéro $(\mathrm{P}<0,05)$.

n'a pas modifié de façon sensible la structure anatomique de la carcasse. Nos résultats permettent d'avoir une idée de l'influence de la sélection réalisée sur les corrélations entre quelques caractéristiques de carcasse, mais il faudrait les compléter par des études sur des effectifs beaucoup plus importants pour obtenir des résultats valables dans ce genre de recherche.

\section{Conclusion}

Le travail de sélection présenté ici a donné des résultats intéressants pour ceux qui cherchent à améliorer les caractéristiques de carcasse du poulet : il est tout à fait possible de diminuer l'état d'engraissement et d'augmenter le rendement en morceaux de premier choix tout en continuant d'améliorer la vitesse de croissance. Les animaux obtenus présentent une meilleure conformation et un meilleur rendement de carcasse pour les utilisateurs.

Il faudrait maintenant étudier si ce type de sélection maintient, ou modifie, d'autres caractéristiques de qualité telles que les performances de reproduction, les défauts d'applomb, les problèmes locomoteurs, la structure et la composition des muscles, ainsi que les caractéristiques organoleptiques et fonctionnelles de la viande de poulet.

\section{Références bibliographiques}

Becker W.A., Spencer J.V., Mirosh L.W., Verstrate J.A., 1984. Genetic variation of abdominal fat, body weight, and carcass weight in a female broiler line. Poultry Sci., 63, 607-611.

Bougon M., Le Menec M., Launay M. Balaine L., 1993. Vingt-sixième épreuve pour reproducteurs de type chair 1991-1992. Sci. Techn. Avic., 2, 4-13.

Chamberlain F.W., 1943. Atlas of Avian Anatomy. Michigan State Coll., Agric. Exp. Station, Memoir Bull. 5 .

Delpech P., Ricard F.H., 1965. Relation entre les dépôts adipeux viscéraux et les lipides corporels chez le poulet. Ann. Zootech., 14, 181-189.

Kazakov A., Friars G.W., Holt J.D., 1984. Relationships of carcass fat and grade traits in broiler chickens. Can. J. Anim. Sci., 64, 613-620.

King S.C., Carson J.R., Doolittle D.P., 1959. The Connecticut and Cornell randombred populations of chickens. World's Poultry Sci. J., 15, 139-159.

Leclercq B., 1988. Genetic selection of meat-type chickens for high or low abdominal fat content. in : Leanness in domestic birds, 25-40. Leclercq and Whitehead Editors, Butterworths, London.

Leclercq B., Blum J.C., Boyer J.P., 1980. Selecting broilers for low or high abdominal fat : initial observations. British Poultry Sci., 21, 107-113.

Leclercq B., Simon J., Ricard F.H., 1987. Effects of selection for high or low plasma glucose concentration in chickens. British Poultry Sci., 28, 557-565.

L'Hospitalier R., Bougon M., Le Menec M. Quemeneur P., 1986. Evolution des performances des poules reproductrices de type-chair et de leur descendance, de 1962 à 1985. Bull. Inf. Sta. Exper. Avic., Ploufragan, 26, 3-16.

Ricard F.H., 1974. Etude de la variabilité génétique de quelques caractéristiques de carcasse en vue de sélectionner un poulet de qualité. Proc. 1st World Cong. Genet. Appl. Livestock Prod., 931-940.

Ricard F.H., Rouvier R., 1967. Etude de la composition anatomique du poulet. Ann. Zootech. $16,23-39$

Ricard F.H., Touraille C., 1988. Selection for leanness and carcass quality. in : Leanness in domestic birds, 377-386. Leclercq and Whitehead Editors, Butterworths, London.

W.P.S.A., Working group V, 1984. Method of dissection of broiler carcases and description of parts. Jensen J. Fris Editor, Pergamon Press, Cambridge, $33 \mathrm{pp}$. 


\section{Summary}

\section{Improvement by selection means of broiler car- cass quality}

Improvement of broiler carcass quality by usual selection techniques was studied during 8 generations in a synthetic line of fast growing chickens. Foundation stock was obtained by crossing several commercial and experimental lines and was then maintained without selection pressure as a random control line. The so-called "quality" line was obtained from this "control" line to give birds with low abdominal fat percentage, high breast meat yield, and body weight as high as possible.

Lowering abdominal fat content was more difficult to obtain than improving breast meat yield because of negative correlations existing between those two characteristics. Nevertheless good results were achieved $:$ in the eighth generation of selection, birds from the selected line were $8 \%$ heavier than control, abdominal fat percentage was $21 \%$ lower, and pectoral muscles percentage was $11 \%$ higher.

Higher breast angles were obtained in selected birds, that means a better carcass conformation. Those birds also showed a lower gizzard percentage, and a higher carcass yield with first choice parts (individual pectoral muscles, drumsticks, wings). Relatively homogeneous values were observed for correlations between carcass parts and yields of the two lines in the eighth generation. This result may be due to the fact that general anatomical structure of the birds was not modified in an appreciable way by the type of selection applied in this experiment.

RICARD F.H., MARCHE G., LE BIHAN-DUVAL E., 1994. Essai d'amélioration par sélection de la qualité de carcasse du poulet de chair. INRA Prod. Anim., 7 (4), $253-261$. 